\title{
Topological color codes on Union Jack lattices: a stable implementation of the whole Clifford group
}

\author{
Helmut G. Katzgraber, ${ }^{1,2}$ H. Bombin, ${ }^{3}$ Ruben S. Andrist, ${ }^{2}$ and M. A. Martin-Delgado ${ }^{4}$ \\ ${ }^{1}$ Department of Physics and Astronomy, Texas A\&M University, College Station, Texas 77843-4242, USA \\ ${ }^{2}$ Theoretische Physik, ETH Zurich, CH-8093 Zurich, Switzerland \\ ${ }^{3}$ Perimeter Institute for Theoretical Physics, Waterloo, Ontario N2L 2Y5, Canada \\ ${ }^{4}$ Departamento de Física Teórica I, Universidad Complutense, 28040 Madrid, Spain
}

(Received 3 October 2009; published 22 January 2010)

\begin{abstract}
We study the error threshold of topological color codes on Union Jack lattices that allow for the full implementation of the whole Clifford group of quantum gates. After mapping the error-correction process onto a statistical mechanical random three-body Ising model on a Union Jack lattice, we compute its phase diagram in the temperature-disorder plane using Monte Carlo simulations. Surprisingly, topological color codes on Union Jack lattices have a similar error stability to color codes on triangular lattices, as well as to the Kitaev toric code. The enhanced computational capabilities of the topological color codes on Union Jack lattices with respect to triangular lattices and the toric code combined with the inherent robustness of this implementation show good prospects for future stable quantum computer implementations.
\end{abstract}

DOI: 10.1103/PhysRevA.81.012319

PACS number(s): 03.67.Lx, 75.40.Mg, 03.67.Pp, 75.50.Lk

\section{INTRODUCTION}

Recently, the error threshold for topological color codes on triangular lattices has been computed using Monte Carlo methods [1], as well as using duality arguments [2]. Understanding the error-correction properties of topological color codes is of paramount importance and serves as a benchmark for comparing to other quantum error-correction codes. Being one of the central figures of merit, error thresholds describe the tolerable value of noise below which a quantum code can perform quantum operations without the impact of decoherence effects due to the environment.

In particular, for topological stabilizer codes there are two main instances of quantum codes that are simple enough such that detailed studies of their computational capabilities can be carried out: the original toric code (TC) introduced by Kitaev [3] and topological color codes (TCC) [4]. In both cases, quantum gates are implemented by external operations that act nontrivially on the degenerate ground-state manifold of a given quantum lattice Hamiltonian. The ground state harbors the topological stabilizer code while excited states correspond to errors in the system. Within this scheme, topological quantum operations are performed based on ground-state properties [5]. Alternatively, it is possible to implement topological quantum gates based on quasiparticle braiding operations [6].

In comparison to the toric code, TCCs can encode a larger number of qubits at a given surface of fixed topology and the variety of topological quantum gates that can be applied transversally is larger [7]. In particular, it is the possibility to perform the whole Clifford group of quantum gates in a transversal and topological way that makes TCCs especially appealing in quantum information theory. The error threshold is one figure of merit for the performance of any topological stabilizer code. If this value is very small for a given code then all the advantages of the new code are meaningless in practical applications because small amounts of external noise would spoil its stability.

Improving the computational capabilities of a quantum information system generally comes at the price of decreased robustness against environmental noise. However, the exact threshold value for the qubit error rate $p$ in TCCs on triangular lattices has been computed numerically to be $p_{\mathrm{c}}=0.109(2)$ [1], which agrees within error bars with the value for the toric code [8-11]. This result-also confirmed by other studies $[2,12,13]$ - is encouraging: Topological color codes preserve a high error tolerance for quantum operations without performance trade-offs (i.e., it is possible to perform complex quantum operations such as quantum distillation, teleportation, and dense coding while retaining the same stability against noise).

To estimate the error tolerance of a TCC, the errorcorrection process is mapped onto a statistical model with random three-body interactions that correspond to the faulty bits. In analogy to the Kitaev model, the random three-body Ising model [1] plays a similar role as the random bond Ising model in the Kitaev toric code [14]. The study of the threebody random Ising model further highlights the relationship between spin-glass physics and quantum information theory, and presents a new class of model systems exhibiting glassy behavior via three-body interactions (i.e., without spin-reversal symmetry).

In this work we revisit the problem and estimate the error threshold for topological color codes on the Union Jack (UJ) lattice. The motivation to use the triangular lattice (which is dual to the hexagonal lattice) in Ref. [1] was based on the triangular lattice being the simplest example of a family of lattices named colexes (color complexes) [15] and the simplicity of numerical simulations. However, there is a technical caveat with TCCs on the hexagonal lattice: they do not fully reproduce the Clifford group [4]. In fact, one of the gate generators of the group needs the square-octagonal lattice for its topological implementation. We therefore use the fact that the UJ lattice is the dual lattice of the square-octagonal lattice.

There is another fundamental reason to study TCCs on the UJ lattice. The presence of three-body random interactions poses new problems that were absent in the Kitaev code. For 
example, the type of lattice and its connectivity may play a role in some of the essential features of the mapped statistical model that is visible in the location of the multicritical point or the values of the critical exponents. To see this, there is another route to establish connections between quantum information and classical statistical mechanical models. It is based on the notion of classical simulability of topological ground states $[16,17]$ and classical models with the completeness property $[18,19]$. The implication of these studies for TCCs is that certain properties of their ground state can be related to a threebody Ising model without randomness $(p=0)$ [17]. Following universality [20,21], it is expected that the critical exponents depend on the lattice geometry and order parameter symmetry $[22,23]$. Therefore, TCCs allow us to see a connection between critical exponents and computational capabilities of a quantum code, and not just the location of its critical point. This is a novel feature that is absent in the statistical mapping from topological codes and random models [14] where only the location of the multicritical point plays a role in the features of the quantum information system. We conjecture that the same connection between lattice-dependent critical exponents and different quantum capabilities for TCCs holds in the presence of randomness $p \neq 0$ at the multicritical point.

Our simulations show that the numerical value of the multicritical point $p_{\mathrm{c}}$ for the three-body Ising model on random UJ lattices agrees within error bars with the value obtained for the triangular lattice [1]. In turn, this highlights the stability of TCCs to external noise. In addition, we compare the phase boundary between a ferromagnetic ground state and a paramagnetic one for the three-body Ising model on both triangular (TR) and UJ lattices. Our results show that these are rather similar.

Note that throughout this article we assume that external error correction is carried out on the protected system represented by the topological color code. This is the standard notion of quantum error correction [24-29]. Recently, experimental realizations of topological error correction have been implemented [30], as well as experimental proposals for TCCs using Rydberg atoms [31] have been made. However, there are also more demanding schemes in which the topological color code can be internally protected leading to the notion of a self-correcting quantum computer [32,33].

The article is organized as follows: In Sec. II we introduce topological color-code states on Union Jack lattices, followed by the mapping of the error-correction process of the TCC onto random three-body Ising models in Sec. III. In Sec. IV we describe our Monte Carlo simulations and how to locate the multicritical point $p_{\mathrm{c}}$ that corresponds to the error threshold of the TCCs. Results from simulations are shown in Sec. V, followed by concluding remarks.

\section{COLOR CODES ON THE UNION JACK LATTICE}

A TCC can be obtained from any two-dimensional (2D) lattice in which all plaquettes are triangles and vertices are three-colorable such that no link connects vertices of the same color. It is also possible to work in the dual lattice (called a two-colex; see Refs. $[4,15]$ ) but here we prefer to work in the triangular lattice to have a more direct mapping, as done in Ref. [1]. The lattice is embedded in a compact surface of

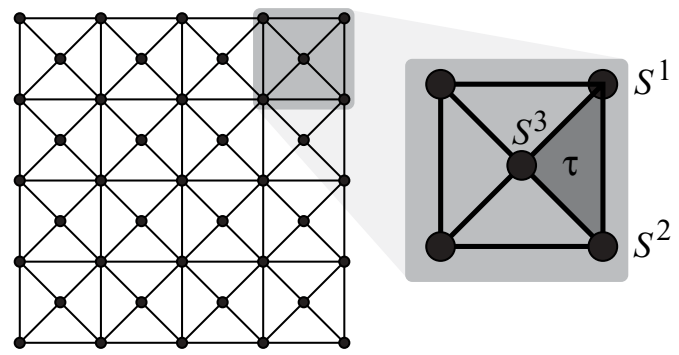

FIG. 1. Simulated Union Jack lattice. The sites at the edges of the squares (inset) have coordination 8, whereas the sites in the center of the square plaquettes have coordination 4. The Hamiltonian in Eq. (2) is a sum over all possible triangles with a weight $\tau$ multiplied by the product of the three spins $S^{1}, S^{2}$, and $S^{3}$ on the triangle's edges (inset). For the simulations, we use periodic boundary conditions (i.e., the lattice is placed on a torus).

arbitrary topology. Since information is encoded in topological degrees of freedom, the code is nontrivial only when the topology of the surface is nontrivial (i.e., the genus $g$ of the surface has to be $g \geqslant 1)$. Note that data can also be encoded in a planar surface with holes and appropriate boundary conditions. This makes topological proposals more amenable to experimental setups [34,35]. In this work we embed the system in a Union Jack lattice (see Fig. 1) to be able to transversally implement all Clifford gates [4].

The construction of the $\operatorname{code} \mathcal{C}$ is done in the following way: Consider a physical system with a qubit at each lattice triangle and introduce the following vertex operators that generate the stabilizer group of $\mathcal{C}$ [28]. For each vertex $v$ there are two types of operators that correspond to Pauli operators of $X$ or $Z$ type:

$$
X_{v}:=\bigotimes_{\triangle: v \in \Delta} X_{\triangle}, \quad Z_{v}:=\bigotimes_{\triangle: v \in \Delta} Z_{\Delta},
$$

that is, a vertex operator acts on all nearby triangles which may be 4 or 8 (see Fig. 1). Vertex operators pairwise commute and square to identity, so that they generate an Abelian group $\mathcal{S}$ called the stabilizer. The code $\mathcal{C}$ is defined as the subspace stabilized by $\mathcal{S}$ and thus contains the states with $X_{v}=Z_{v}=$ $1 \forall v$.

What makes the UJ lattice special is that the vertex operators - the generators of the stabilizer-have support on a number of qubits that is a multiple of four. This fact, together with the properties of general color codes, gives rise to an important feature, namely that Clifford gates leave the stabilizer invariant. After the introduction of suitable boundaries on the lattice, this allows for the transversal implementation of the Clifford group on color codes [4].

\section{ERROR-CORRECTION MAPPING}

When an encoded state in a color $\operatorname{code} \mathcal{C}$ is subject to errors, the first step for their possible correction is the measurement of vertex operators. The resulting collection of \pm 1 eigenvalues is called the error syndrome and gives information about which errors occurred. Indeed, the errors need not be guessed exactly but only up to a stabilizer element.

Color codes have a structure with stabilizer generators that are either products of $X$ or $Z$ Pauli operators, but not both. This allows us to treat bit-flip and phase errors 
separately: $X$-type ( $Z$-type) errors produce violations of $Z$-type ( $X$-type) vertex operators. The correction of each type of error can be expressed in homological terms (see Ref. [1]) such that the syndrome gives the boundary of the error. In order to do a successful correction, errors must be guessed only up to homology. In contrast to toric codes where one deals with the usual homology of paths on a surface, in TCCs two different types (colors) of paths are allowed.

Consider a standard error model based on stochastic errors in which phase errors $Z$ and bit-flip errors $X$ are uncorrelated and occur with probability $p$ at each qubit. In topological codes, when $p$ is below a threshold $p_{\mathrm{c}}$, error correction can be performed with perfect accuracy in the limit of infinite system size. Above the threshold, error correction becomes useless in the same limit. In Ref. [14] this error threshold was computed for toric codes by mapping the error-correction process to a random bond Ising model. The corresponding mapping for color codes was carried out in Ref. [1], which showed that a random three-body Ising model appears. Note that other general extensions of statistical mappings are also possible in the context of topological subsystem codes [36].

Let us summarize what happens when we apply the mapping to the Union Jack lattice. First, the spins of the statistical model correspond to the stabilizer generators. Thus, we have a classical spin at each vertex of the lattice. As for the three-body interactions, they correspond to the physical qubits and hence there is such a term per triangle. Each of these terms carries a random sign yielding the Hamiltonian

$$
\mathcal{H}=-J \sum_{\triangle} \tau_{\triangle} S_{\triangle}^{1} S_{\triangle}^{2} S_{\triangle}^{3},
$$

where the coupling $J$ is positive and the sum is over the product of the spins at the vertices of all triangles on the UJ lattice and $\tau_{\Delta}= \pm 1$ [37]. The (classical) Ising spins $S_{\Delta}^{i}$ can have the values \pm 1 . The sign of the coupling constants $\tau$ are independent random quenched variables, that are negative with probability $p$.

At low temperature $T$ and small disorder $p$, the system is ferromagnetic. Above a critical line $p_{\text {crit }}(T)$, the model undergoes a phase transition to a paramagnetic state. The error threshold $p_{\mathrm{c}}$ is given by the crossing point between $p_{\text {crit }}(T)$ and the Nishimori line $[38,39]$

$$
\exp (-2 J / T)=\frac{p}{(1-p)}
$$

\section{NUMERICAL DETAILS}

When studying phase transitions of statistical systems, it is most favorable to study quantities whose finite-size scaling form is dimensionless (i.e., there is not a system-size dependent prefactor in front of the scaling function). Typical quantities are the Binder ratio [40] as well as the dimensionless twopoint finite-size correlation length divided by the system size [41]. The latter has been shown to be a more robust measure of transition temperatures for systems without spin-reversal symmetry [1] because the Binder ratio becomes negative and steep at the transition (i.e., pinpointing the critical temperature is difficult).
The transitions to a ferromagnetic phase are determined by a finite-size scaling of the dimensionless two-point finite-size correlation length divided by the system size. We start by determining the wave-vector-dependent susceptibility

$$
\chi(k)=\frac{1}{L^{2}} \sum_{i j}^{N}\left\langle S_{i} S_{j}\right\rangle_{T} e^{i \mathbf{k} \cdot\left(\mathbf{R}_{i}-\mathbf{R}_{j}\right)} .
$$

In Eq. (4) $\langle\cdots\rangle_{T}$ represents a thermal average and $\mathbf{R}_{i}$ the spatial location of the spins. The correlation length is then given by

$$
\xi_{\mathrm{m}}=\frac{1}{2 \sin \left(k_{\min } / 2\right)} \sqrt{\frac{[\chi(k=0)]_{\mathrm{av}}}{\left[\chi\left(k_{\min }\right)\right]_{\mathrm{av}}}-1},
$$

where $k_{\min }=(2 \pi / L, 0)$ is the smallest nonzero wave vector and $[\cdots]_{\mathrm{av}}$ represents an average over the different error configurations (disorder sampling). The finite-size correlation length divided by the system size has the following finite-size scaling form:

$$
\xi_{\mathrm{m}} / L \sim \widetilde{X}\left(L^{1 / v}\left[T-T_{\mathrm{c}}\right]\right)
$$

where $v$ is a critical exponent and $T_{\mathrm{c}}$ represents the transition temperature. Numerically, finite systems of linear size $L$ are studied. In that case the function $\xi_{\mathrm{m}} / L$ is independent of $L$ whenever $T=T_{\mathrm{c}}$ as then the argument of the function $\tilde{X}$ is zero. In other words, when different system sizes are studied, the data cross at one point, which corresponds to the transition, if present. This can be seen in Fig. 2. Because finite-size scaling corrections are small in this case, one can use the estimate of $T_{\mathrm{c}}$ obtained as a very good approximation to the thermodynamic limit value. The critical exponent $v$ for the correlation length can be determined by a full scaling of the data [42], as shown in Ref. [1].

We have also computed the spin-glass finite-size correlation length [replace $S_{i}$ with $S_{i}^{\alpha} S_{i}^{\beta}$ in Eq. (4)]. For all $p>p_{\text {c }}$ studied, no sign of a finite-temperature spin-glass transition was found. This result coincides with the results found for the triangular lattice [1] and is compatible with the standard belief [39] that no glassy phase is expected in two-dimensional random models, although here this is extended to systems with three-body interactions. We believe that a spin-glass phase may be possible for spin models with random many-body interactions in three space dimensions.

Because the complexity of the problem increases considerably when $p>0$, we use parallel tempering Monte Carlo [43,44]. In addition to local spin flips, after each lattice sweep a global update that exchanges the temperature of two replicas (copies) of the system is proposed. This considerably speeds up the simulations (parameters are shown in Table I). Equilibration is tested by a logarithmic binning of the data. Once at least the last three bins agree within errors, we define the system to be equilibrated. Note that we use periodic boundary conditions to reduce finite-size effects. To prevent a mismatch with the vertex coloring rules of the lattice and boundary conditions, we use system sizes $L$ that are a multiple of 6 .

\section{RESULTS}

Figure 2 shows the temperature-dependent dimensionless finite-size correlation length for different values of $p$. The 

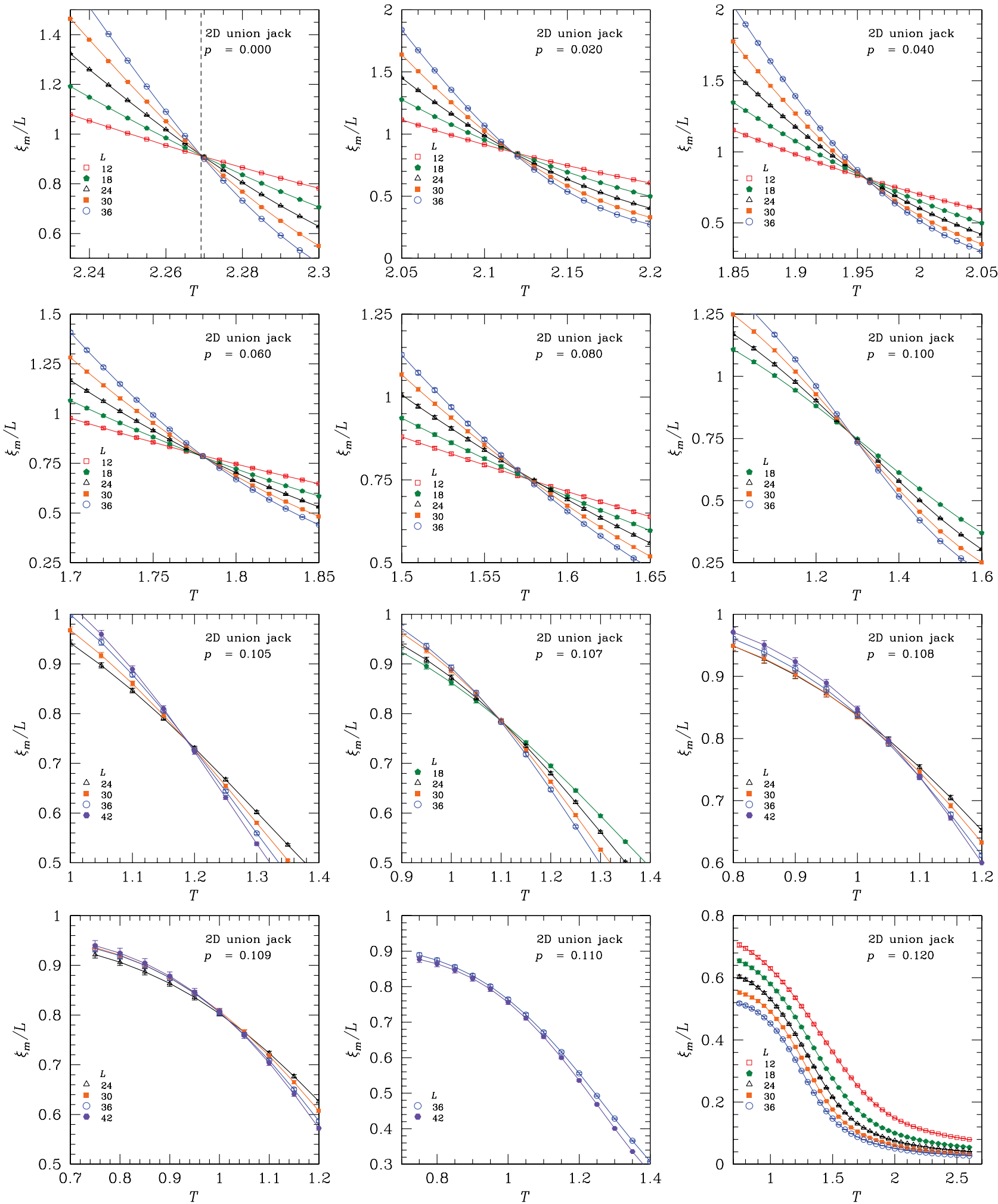

FIG. 2. (Color online) Finite-size correlation length $\xi_{m} / L$ as a function of temperature $T$ for different values of $p$. The data for $p=0$ cross at the critical temperature of the 2D Ising model (dashed line, top left panel). For $p \lesssim p_{\mathrm{c}}=0.109$ there is signature of a transition (data for different $L$ cross) whereas for $p=0.110>p_{\mathrm{c}}$ the transition vanishes. Note that for $p \gtrsim 0.108$, corrections are large thus making the determination of the transition temperature difficult. For the case of $p=0.110$ we only show the two largest sizes for clarity.

top-left panel shows data for $p=0$. The vertical dashed line is the analytically known transition temperature of the two-dimensional Ising model (i.e., $T_{\mathrm{c}} \simeq 2.2692$ [20]). It is remarkable that the three-body Ising model on the Union
Jack lattice has the same transition temperature, albeit being in a different universality class. Its critical exponents are fully determined by knowing two of them (e.g., $v=3 / 4$ and $\alpha=1 / 2$ [45]). For $p \approx 0.108$ critical behavior sets in, and for 
TABLE I. Simulation parameters: $L$ is the system size, $N_{\mathrm{sa}}$ is the number of disorder samples, $t_{\mathrm{eq}}=2^{b}$ is the number of equilibration sweeps, $T_{\min }\left(T_{\max }\right)$ is the lowest (highest) temperature, and $N_{\mathrm{T}}$ the number of temperatures used.

\begin{tabular}{|c|c|c|c|c|c|c|}
\hline$p$ & $L$ & $N_{\mathrm{sa}}$ & $b$ & $T_{\min }$ & $T_{\max }$ & $N_{\mathrm{T}}$ \\
\hline 0.00 & 12,18 & 50 & 18 & 2.200 & 2.350 & 31 \\
\hline 0.00 & 24,30 & 50 & 19 & 2.200 & 2.350 & 31 \\
\hline 0.00 & 36 & 50 & 20 & 2.200 & 2.350 & 31 \\
\hline 0.01 & 12,18 & $5 \times 10^{3}$ & 18 & 1.900 & 2.400 & 51 \\
\hline 0.01 & 24,30 & $5 \times 10^{3}$ & 19 & 1.900 & 2.400 & 51 \\
\hline 0.01 & 36 & $5 \times 10^{3}$ & 20 & 1.900 & 2.400 & 51 \\
\hline 0.02 & 12,18 & $5 \times 10^{3}$ & 18 & 1.900 & 2.400 & 51 \\
\hline 0.02 & 24,30 & $5 \times 10^{3}$ & 19 & 1.900 & 2.400 & 51 \\
\hline 0.02 & 36 & $5 \times 10^{3}$ & 20 & 1.900 & 2.400 & 51 \\
\hline 0.03 & 12,18 & $5 \times 10^{3}$ & 18 & 1.700 & 2.200 & 51 \\
\hline 0.03 & 24,30 & $5 \times 10^{3}$ & 19 & 1.700 & 2.200 & 51 \\
\hline 0.03 & 36 & $5 \times 10^{3}$ & 20 & 1.700 & 2.200 & 51 \\
\hline 0.04 & 12,18 & $5 \times 10^{3}$ & 18 & 1.700 & 2.200 & 51 \\
\hline 0.04 & 24,30 & $5 \times 10^{3}$ & 19 & 1.700 & 2.200 & 51 \\
\hline 0.04 & 36 & $5 \times 10^{3}$ & 20 & 1.700 & 2.200 & 51 \\
\hline 0.06 & 12,18 & $5 \times 10^{3}$ & 18 & 1.600 & 2.100 & 51 \\
\hline 0.06 & 24,30 & $5 \times 10^{3}$ & 19 & 1.600 & 2.100 & 51 \\
\hline 0.06 & 36 & $5 \times 10^{3}$ & 20 & 1.600 & 2.100 & 51 \\
\hline 0.08 & 12,18 & $5 \times 10^{3}$ & 18 & 1.400 & 2.000 & 61 \\
\hline 0.08 & 24,30 & $5 \times 10^{3}$ & 19 & 1.400 & 2.000 & 61 \\
\hline 0.08 & 36 & $5 \times 10^{3}$ & 20 & 1.400 & 2.000 & 61 \\
\hline $0.10-0.11$ & 12,18 & $10^{4}$ & 18 & 0.750 & 2.600 & 38 \\
\hline $0.10-0.11$ & 24,30 & $10^{4}$ & 19 & 0.750 & 2.600 & 38 \\
\hline $0.10-0.11$ & 36 & $10^{4}$ & 20 & 0.750 & 2.600 & 38 \\
\hline $0.10-0.11$ & 42 & $10^{4}$ & 22 & 0.750 & 2.600 & 38 \\
\hline 0.12 & 12,18 & $5 \times 10^{3}$ & 18 & 0.750 & 2.600 & 38 \\
\hline 0.12 & 24,30 & $5 \times 10^{3}$ & 19 & 0.750 & 2.600 & 38 \\
\hline 0.12 & 36 & $5 \times 10^{3}$ & 20 & 0.750 & 2.600 & 38 \\
\hline
\end{tabular}

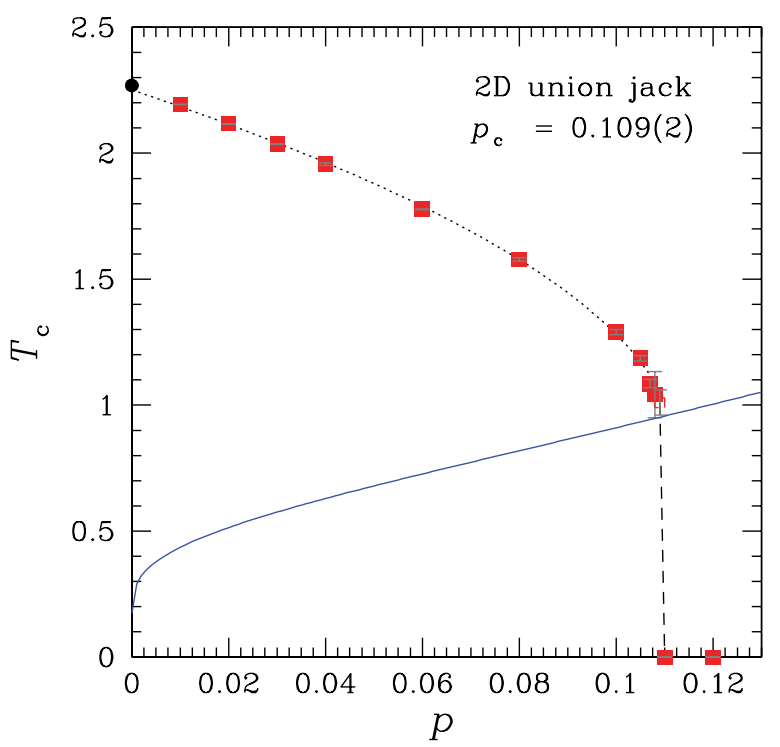

FIG. 3. (Color online) $p-T_{\mathrm{c}}$ phase diagram for the random threebody Ising model on the Union Jack lattice. For $p>p_{\mathrm{c}} \approx 0.109$ the ferromagnetic order is lost. The dotted line is a guide to the eye, the black circle represents the analytically known transition temperature of the 2D Ising model. The solid line represents the Nishimori line. In the regime marked by a dashed line the exact determination of $T_{\mathrm{c}}(p)$ is difficult.

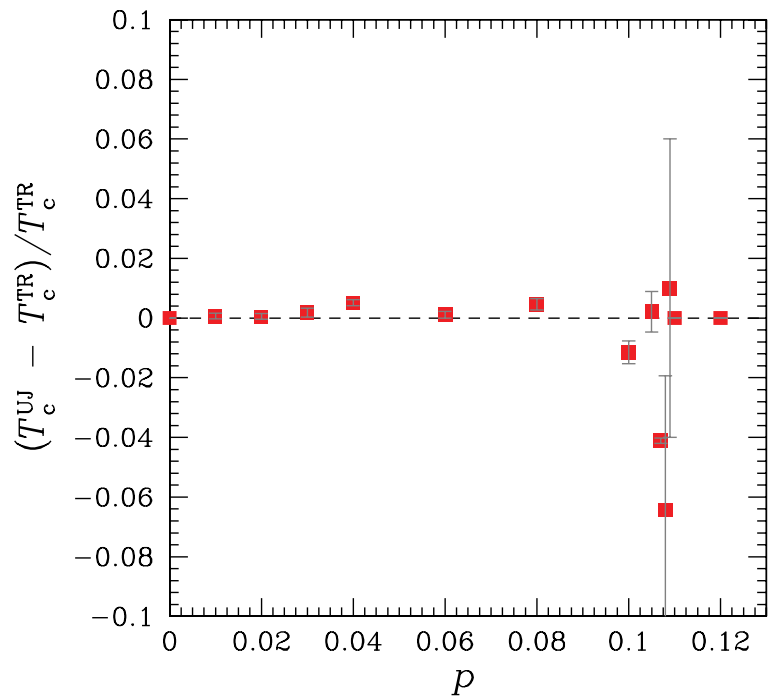

FIG. 4. (Color online) Relative difference between $T_{\mathrm{c}}$ for the Union Jack (UJ) and triangular lattices (TR) [1] as a function of $p$. In most cases the deviations are smaller than $1 \%$. Around $p_{\mathrm{c}} \approx 0.109$ strong fluctuations appear since it is hard to estimate the transition temperatures. The data for the triangular lattice have been adapted from Ref. [1] and expanded. Note that for $p \rightarrow 0$ the deviations are smallest, suggesting that the slopes of the phase lines for $p=0$ are very close.

$p=0.110$ no sign of a transition is visible. We thus estimate $p_{\mathrm{c}}=0.109(2)$, in agreement with results for the triangular (TR) lattice [1] and the toric code [8-11].

The full $p-T_{\mathrm{c}}$ phase diagram for the UJ lattice is shown in Fig. 3; the solid line is the Nishimori line. To check the differences between the phase diagram for the UJ and TR lattices, we plot the relative deviation between the different estimates of the critical temperature, $\left(T_{\mathrm{c}}^{\mathrm{UJ}}-T_{\mathrm{c}}^{\mathrm{TR}}\right) / T_{\mathrm{c}}^{\mathrm{TR}}$, as a function of $p$ (Fig. 4). For most values of $p$ the fluctuations are statistical and not larger than $1 \%$. For $p \sim p_{\mathrm{c}}$, fluctuations are larger and the deviations are of the order of $\sim 7 \%$. Therefore, we believe that the phase boundaries for both models are very close.

\section{CONCLUSIONS}

In this work we have computed the $p-T$ phase diagram for the random three-body Ising model on a two-dimensional Union Jack lattice (Figs. 1 and 3). The original motivation for this numerical study with Monte Carlo methods is to compute the multicritical point $p_{\mathrm{c}}$ in this phase diagram: the crossing point between the critical line separating ordered/disordered phases and the Nishimori line. The crossing point $p_{\mathrm{c}}$ corresponds to the error threshold for topological color codes defined on the square-octagonal lattice (the dual of which is the Union Jack lattice) — a value that decides whether a topological stabilizer code is good enough for performing quantum error correction in practical applications. Our numerical result of $p_{\mathrm{c}}=0.109(2)$ is in agreement with the corresponding value for the TCCs on triangular lattices and the toric code, within error bars. This result shows that TCCs on the square-octagonal lattice, which allow for the implementation of the complete Clifford group of quantum gates [4], are similarly stable as the toric code. The fact that the triangular lattice and the UJ 
lattice share similar values of $p_{\mathrm{c}}$ that agree within error bars is by no means obvious since both models are known to be in different universality classes. Note that the Monte Carlo method combined with finite-size scaling used to determine the error threshold does not rely on approximations. The computed values of the phase boundary can merely be affected by finite system sizes, although corrections to scaling are small. Furthermore, effects of realistic error models have yet to be tested.

\section{ACKNOWLEDGMENTS}

We thank A. Landahl and M. Ohzeki for useful discussions. The authors acknowledge Texas A\&M University for access to their hydra cluster, the Texas Advanced Computing Center (TACC) at The University of Texas at Austin for providing HPC resources (Ranger Sun Constellation Linux Cluster), the Centro de Supercomputación y Visualización de Madrid (CeSViMa) for access to the magerit cluster, the Barcelona Supercomputing Center for access to the MareNostrum cluster within the Spanish Supercomputing Network, and ETH Zurich for CPU time on the Brutus cluster. M.A.M.-D. and H.B. acknowledge financial support from a PFI grant of EJ-GV, from DGS Grants under Contract Nos. FIS2006-04885 and FIS2009-10061 from the ESF INSTANS 2005-10. H.G.K. acknowledges support from the SNF (Grant No. PP002114713).
[1] H. G. Katzgraber, H. Bombin, and M. A. Martin-Delgado, Phys. Rev. Lett. 103, 090501 (2009).

[2] M. Ohzeki, Phys. Rev. E 80, 011141 (2009).

[3] A. Y. Kitaev, Ann. Phys. 303, 2 (2003).

[4] H. Bombin and M. A. Martin-Delgado, Phys. Rev. Lett. 97, 180501 (2006).

[5] H. Bombin and M. A. Martin-Delgado, Phys. Rev. Lett. 98, 160502 (2007).

[6] C. Nayak, S. H. Simon, A. Stern, M. Freedman, and S. Das Sarma, Rev. Mod. Phys. 80, 1083 (2008).

[7] H. Bombin and M. A. Martin-Delgado, Phys. Rev. A 76, 012305 (2007).

[8] A. Honecker, M. Picco, and P. Pujol, Phys. Rev. Lett. 87, 047201 (2001).

[9] F. Merz and J. T. Chalker, Phys. Rev. B 65, 054425 (2002).

[10] M. Ohzeki, Phys. Rev. E 79, 021129 (2009).

[11] F. Parisen Toldin, A. Pelissetto, and E. Vicari, J. Stat. Phys. 135, 1039 (2009).

[12] D. S. Wang, A. G. Fowler, C. D. Hill, and L. C. L. Hollenberg, e-print arXiv:0907.1708.

[13] A. Landahl, J. T. Anderson, and P. Rice (in preparation, 2009).

[14] E. Dennis, A. Kitaev, A. Landahl, and J. Preskill, J. Math. Phys. 43, 4452 (2002).

[15] H. Bombin and M. A. Martin-Delgado, Phys. Rev. B 75, 075103 (2007).

[16] S. Bravyi and R. Raussendorf, Phys. Rev. A 76, 022304 (2007).

[17] H. Bombin and M. A. Martin-Delgado, Phys. Rev. A 77, 042322 (2008).

[18] M. Van den Nest, W. Dür, and H. J. Briegel, Phys. Rev. Lett. 100, 110501 (2008).

[19] G. de Las Cuevas, W. Dür, H. J. Briegel, and M. A. MartinDelgado, Phys. Rev. Lett. 102, 230502 (2009).

[20] J. M. Yeomans, Statistical Mechanics of Phase Transitions (Oxford University Press, Oxford, 1992).

[21] J. Cardy, Scaling and Renormalization in Statistical Physics (Cambridge University Press, Cambridge, 1996).

[22] A. Hintermann and D. Merlini, Phys. Lett. A41, 208 (1972).

[23] R. J. Baxter and F. Y. Wu, Phys. Rev. Lett. 31, 1294 (1973).
[24] P. W. Shor, Phys. Rev. A 52, R2493 (1995).

[25] A. M. Steane, Phys. Rev. Lett. 77, 793 (1996).

[26] A. R. Calderbank and P. W. Shor, Phys. Rev. A 54, 1098 (1996).

[27] A. Steane, Proc. R. Soc. London A 452, 2551 (1996).

[28] D. Gottesman, Phys. Rev. A 54, 1862 (1996).

[29] H. Bombin and M. A. Martin-Delgado, J. Math. Phys. 48, 052105 (2007)

[30] W.-B. Gao et al., e-print arXiv:0905.1542.

[31] H. Weimer, M. Müller, I. Lesanovsky, P. Zoller, and H. P. Büchler, e-print arXiv:0907.1657.

[32] H. Bombin, R. W. Chhajlany, M. Horodecki, and M. A. MartinDelgado, e-print arXiv:0907.5228.

[33] R. Alicki, M. Horodecki, P. Horodecki, and R. Horodecki, e-print arXiv:0811.0033 [quant-ph].

[34] S. Bravyi and A. Y. Kitaev, e-print arXiv:quant-ph/9811052.

[35] H. Bombin and M. A. Martin-Delgado, J. Phys. A 42, 095302 (2009).

[36] H. Bombin, e-print arXiv:0908.4246.

[37] Without loss of generality, we set the energy scale $J=1$. Therefore, temperatures are dimensionless.

[38] H. Nishimori, Prog. Theor. Phys. 66, 1169 (1981).

[39] H. Nishimori, Statistical Physics of Spin Glasses and Information Processing: An Introduction (Oxford University Press, New York, 2001).

[40] K. Binder and A. P. Young, Rev. Mod. Phys. 58, 801 (1986).

[41] M. Palassini and S. Caracciolo, Phys. Rev. Lett. 82, 5128 (1999).

[42] A finite-size scaling of the data for $p=0$ using the known exponents $v=3 / 4(\alpha=1 / 2)$ shows that corrections to scaling are small.

[43] C. Geyer, in 23rd Symposium on the Interface, edited by E. M. Keramidas (Interface Foundation, Fairfax Station, 1991), p. 156.

[44] K. Hukushima and K. Nemoto, J. Phys. Soc. Jpn. 65, 1604 (1996).

[45] For the two-dimensional Ising ferromagnet on the square lattice $v=1$ and $\alpha=0$. Note that for the three-body Ising model on the triangular lattice $v=\alpha=2 / 3$ [46].

[46] R. Baxter, Exactly Solved Models in Statistical Mechanics (Academic Press, London, 1982). 